\title{
Comparative Analysis of Machine Learning Algorithms for Multi-Syndrome Classification of Neurodegenerative Syndromes
}

Leonie Lampe ( $\sim$ leonie.lampe@aicura-medical.com )

AICURA medical $\mathrm{GmbH}$

\section{Sebastian Niehaus}

AlCURA medical GmbH https://orcid.org/0000-0003-1291-6836

Hans-Jürgen Huppertz

Swiss Epilepsy Clinic, Klinik Leng

Alberto Merola

AICURA medical GmbH

Janis Reinelt

AICURA medical GmbH

\section{Karsten Mueller}

Max Planck Institute for Human Cognitive and Brain Sciences: Max-Planck-Institut fur Kognitions- und Neurowissenschaften

\section{Sarah Anderl-Straub}

Department of Neurology, University of Ulm

\section{Klaus Fassbender}

Department of Neurology, University Saarland

\section{Klaus Fliessbach}

Universitätsklinikum Bonn: Universitatsklinikum Bonn

\section{Holger Jahn}

Clinic for Psychiatry and Psychotherapy, Universitätsklinikum Hamburg-Eppendorf: Universitatsklinikum Hamburg-Eppendorf

\section{Johannes Kornhuber}

Erlangen University Hospital: Universitatsklinikum Erlangen

\section{Martin Lauer}

Universitätsklinikum Würzburg: Universitatsklinikum Wurzburg

Johannes Prudlo

Rostock University Medical Center: Universitatsmedizin Rostock

\section{Anja Schneider}

Universitätsklinikum Bonn: Universitatsklinikum Bonn

\section{Matthis Synofzik}


University of Tuebingen

\section{Adrian Danek}

Ludwig-Maximilians-Universität München: Ludwig-Maximilians-Universitat Munchen

\section{Janine Diehl-Schmid}

TU München: Technische Universitat Munchen

\section{Markus Otto}

Ulm University: Universitat Ulm

\section{Arno Villringer}

Max Planck Institute for Human Cognitive and Brain Sciences Department of Neurophysics: Max-

Planck-Institut fur Kognitions- und Neurowissenschaften

\section{Karl Egger}

Freiburg University Hospital: Universitatsklinikum Freiburg

\section{Elke Hattingen}

Universitätsklinikum Bonn: Universitatsklinikum Bonn

\section{Rüdiger Hilker-Roggendorf}

Klinikum Vest GmbH Behandlungszentrum Knappschaftskrankenhaus Recklinghausen

\section{Alfons Schnitzler}

Heinrich Heine University Düsseldorf: Heinrich-Heine-Universitat Dusseldorf

\section{Martin Südmeyer}

Ernst von Bermann Klinikum, Potsdam

\section{Wolfgang Oertel}

Philips-University Marburg

\section{Jan Kassubek}

Ulm University: Universitat Ulm

\section{Günther Höglinger}

Munich University of Technology: Technische Universitat Munchen

\section{Matthias L. Schroeter}

Max Planck Institute for Human Cognitive and Brain Sciences: Max-Planck-Institut fur Kognitions- und Neurowissenschaften

\section{Research}

Keywords: Machine Learning Algorithms, Multi-Syndrome Classification, Neurodegenerative Syndromes, Comparative Analysis

Posted Date: February 4th, 2021

DOI: https://doi.org/10.21203/rs.3.rs-178965/v1 
License: (c) (i) This work is licensed under a Creative Commons Attribution 4.0 International License. Read Full License 


\section{Comparative Analysis of Machine Learning Algorithms for Multi-Syndrome Classification of Neurodegenerative Syndromes}

Leonie Lampe, MD ${ }^{1,2,3 *}$; Sebastian Niehaus, M.Sc. ${ }^{1,4 *}$; Hans-Jürgen Huppertz, MD ${ }^{5}$; Alberto Merola, $\mathrm{PhD}^{1}$; Janis Reinelt ${ }^{1}$; Karsten Mueller, $\mathrm{PhD}^{3}$; Sarah Anderl-Straub, $\mathrm{PhD}^{6}$; Klaus Fassbender, $\mathrm{MD}^{7}$; Klaus Fliessbach, $\mathrm{MD}^{8}$; Holger Jahn, $\mathrm{MD}^{9}$; Johannes Kornhuber, $\mathrm{MD}^{10}$; Martin Lauer, MD ${ }^{11}$; Johannes Prudlo, MD ${ }^{12}$; Anja Schneider, $\mathrm{MD}^{8}$; Matthis Synofzik, MD ${ }^{13}$; Adrian Danek, MD ${ }^{14}$; Janine Diehl-Schmid, MD ${ }^{15}$; Markus Otto, MD ${ }^{6}$; FTLD-Consortium Germany; Arno Villringer, MD ${ }^{2,3}$; Karl Egger, $\mathrm{MD}^{16}$; Elke Hattingen, $\mathrm{MD}^{17}$; Rüdiger HilkerRoggendorf, MD ${ }^{18}$; Alfons Schnitzler, MD ${ }^{19}$; Martin Südmeyer, MD ${ }^{19,20}$; Wolfgang Oertel, $\mathrm{MD}^{21}$; German Atypical Parkinson Consortium Study Group; Jan Kassubek, MD ${ }^{6}$; Günter Höglinger, $\mathrm{MD}^{22}$; Matthias L Schroeter, $\mathrm{MD} \mathrm{PhD}{ }^{2,3}$

${ }^{1}$ AICURA medical GmbH, Berlin

${ }^{2}$ Clinic for Cognitive Neurology, University Clinic Leipzig, Germany

${ }^{3}$ Max Planck Institute for Human Cognitive and Brain Sciences, Leipzig, Germany

${ }^{4}$ Carl Gustav Carus Faculty of Medicine, Institute for Medical Informatics and Biometry, TU Dresden, Dresden, Germany

${ }^{5}$ Swiss Epilepsy Clinic, Klinik Lengg, Zurich, Switzerland

${ }^{6}$ Department of Neurology, University of Ulm, Germany

${ }^{7}$ Department of Neurology, Saarland University, Homburg, Germany

${ }^{8}$ Clinic for Neurodegenerative Diseases and Geriatric Psychiatry, University of Bonn, and German Center for Neurodegenerative Diseases (DZNE), Bonn, Germany

${ }^{9}$ Clinic for Psychiatry and Psychotherapy, University Hospital Hamburg-Eppendorf, Germany

${ }^{10}$ Department of Psychiatry and Psychotherapy, Friedrich-Alexander-University of Erlangen-Nuremberg, Erlangen, Germany

${ }^{11}$ Department of Psychiatry and Psychotherapy, University Wuerzburg, Germany

${ }^{12}$ Department of Neurology, University of Rostock, and DZNE, Rostock, Germany

${ }^{13}$ Department of Neurodegenerative Diseases, Centre for Neurology \& Hertie-lnstitute for Clinical Brain Research, University of Tuebingen, Germany \& DZNE, Tuebingen, Germany

${ }^{14}$ Department of Neurology, Ludwig-Maximilians-Universität München, Germany

${ }^{15}$ Department of Psychiatry and Psychotherapy, Technical University of Munich, Germany

${ }^{16}$ Department of Neuroradiology, University Hospital of Freiburg, Germany

${ }^{17}$ Department of Neuroradiology, University Hospital of Frankfurt, Germany

${ }^{18}$ Department of Neurology, Klinikum Vest, Recklinghausen, Germany

${ }^{19}$ Institute of Clinical Neurosciences and Medical Psychology, Heinrich Heine University of Düsseldorf, Germany

${ }^{20}$ Department of Neurology, Ernst von Bergmann Klinikum, Potsdam, Germany

${ }^{21}$ Department of Neurology, Philips-University Marburg, Germany

${ }^{22}$ Department of Neurology, Technical University of Munich, Germany; German Center for Neurodegenerative Diseases, Munich, Germany

*shared first authorship

Corresponding author:

1. Prof. Matthias L. Schroeter, schroet@ cbs.mpg.de, +49 3419724962

2. Dr. Leonie Lampe, leonie.lampe@ @aicura-medical.com, +49 6221162492 


\section{Key Points}

Question: This study compares different machine learning methods for predicting several neurodegenerative syndromes.

Findings: The comparison of support vector machine, random forest, gradient boosting, and deep feed-forward neural networks yielded the neural networks to be best for the classification of different neurodegenerative syndromes based on pre-structured volume measures.

Meaning: Even with pre-structured data, deep neural networks are most promising. 


\begin{abstract}
Importance The entry of artificial intelligence into medicine is pending. Several methods have been used for predictions of structured neuroimaging data, yet nobody compared them in this context.
\end{abstract}

Objective Multi-class prediction is key for building computational aid systems for differential diagnosis. We compared support vector machine, random forest, gradient boosting, and deep feed-forward neural networks for the classification of different neurodegenerative syndromes based on structural magnetic resonance imaging.

Design, Setting, and Participants Atlas-based volumetry was performed on multi-centric T1-weighted MRI data from 940 subjects, i.e. 124 healthy controls and 816 patients with ten different neurodegenerative diseases, leading to a multi-diagnostic multi-class classification task with eleven different classes.

\title{
Interventions n.a.
}

Main Outcomes and Measures Cohen's Kappa, Accuracy, and F1-score to assess model performance.

Results Over all, the neural network produced both the best performance measures as well as the most robust results. The smaller classes however were better classified by either the ensemble learning methods or the support vector machine, while performance measures for small classes were comparatively low, as expected. Diseases with regionally specific and pronounced atrophy patterns were generally better classified than diseases with wide-spread and rather weak atrophy. Conclusions and Relevance Our study furthermore underlines the necessity of larger data sets but also calls for a careful consideration of different machine learning methods that can handle the type of data and the classification task best.

\section{Trial Registration n.a.}




\section{Introduction}

In light of the demographic shift and the pending shortage of resources in healthcare systems across the globe, computer aided methods are to shoulder some of the challenges. Supportive technology will find its way into the clinic to assist physicians in finding the correct diagnosis. ${ }^{1}$ The implementation of artificial intelligence into clinical routine is happening already and it is a matter of time until medical decisions will rely on algorithms in conjunction with the experience of physicians.

In case of neurodegenerative syndromes, brain imaging can render important MRI-morphological biomarkers in form of atrophy patterns. While some focal atrophy patterns are quite

disease-specific ${ }^{2,3,4,5,6,7,8}$ leading even to incorporation into diagnostic criteria, ${ }^{9,10,11}$ neuroimaging findings for other diseases might be less conclusive. ${ }^{12}$ However, it requires highly trained and specialized neuroradiologists to correctly detect and interpret the signs - an expertise that is not available ubiquitously.

For analyzing the complex multivariate and non-linear relationships in high dimensional data derived from MRI data, machine learning algorithms are superior to standard inferential statistics. ${ }^{13,14}$ For the classification of neurological and psychiatric diseases, support vector machines (SVM) based on imaging-derived data have been the most popular method. ${ }^{14} \mathrm{SVMs}$ have proven to be a suitable approach at least in binary differentiations of patients from healthy controls. ${ }^{14,13,15}$ A few studies further used SVM to differentiate disease entities from each othera more complex approach that simulates the process of differential diagnosis. In a previous study, we assessed the performance of SVM to differentiate two dementia syndromes from each other. ${ }^{16,17}$ In another study, SVM was used to classify various parkinsonian syndromes based on 
the results of volumetric MRI analysis. ${ }^{18}$ While SVM produced satisfactory results, other methodological approaches were not assessed further.

In recent years deep learning methods have become more and more popular for pattern recognition tasks such as the classification of image and text data, but also of structured data. ${ }^{19}$ Deep learning methods process data on several levels. In this way more and more abstract representations are generated up to the class as the most abstract form of representation. ${ }^{19}$ Deep neural networks (DNN) in particular have proven to be highly proficient in predicting diagnoses based on imaging data of the eye, skin or lung $20,21,22$ and will most likely become a key component of imaging diagnostics in the future. Hopefully, these advanced models will be able to capture more complex atrophy patterns in the human brain than SVM approaches and might assist radiologists with their assessment in the future.

Accordingly, we will compare these models for the classification of neurodegenerative syndromes based on atlas-based volume measures in a very large dataset including numerous diseases in this work. Beside DNN and SVM we will apply two ensemble learning methods (i.e., random forest $(\mathrm{RF})$ and gradient boosting $(\mathrm{GB})$ ) that have been thriving as proficient models in many classification challenges dealing with similar data. ${ }^{14,23}$ The preprocessing of the data into structured data via volumetry in the form of an atlas is useful for clinical purposes, because it normalizes data, reduces thereby inter-center variability, guarantees a complete anonymization of the data and decreases computing time when training the models.

The syndromes considered in this study all belong to the neurodegenerative disease spectrum ranging from Alzheimer's disease $(\mathrm{AD})$, frontotemporal lobar degeneration with its subtypes behavioral variant frontotemporal dementia (bvFTD), and primary progressive aphasias (PPA) with the three subforms - semantic variant (svPPA), nonfluent-agrammatic variant (nfvPPA), and 
logopenic variant (lvPPA) - to atypical Parkinson syndromes such as corticobasal syndrome (CBS), progressive supranuclear palsy (PSP), multiple system atrophy with cerebellar features (MSA-C), as well as MSA with predominant parkinsonism (MSA-P), and idiopathic Parkinson's disease (PD).

This use case is exemplary for imaging-derived structural data and can be transferred to other use cases of the biomedical sciences. By including ten different neurodegenerative diseases beside a control cohort our approach mirrors best the work of radiologists in clinical routine, i.e. firstly categorizing a brain scan as normal or abnormal, and secondly, defining the neurodegenerative entity in the differential diagnostic process. We hypothesize (i) that neurodegenerative diseases can be classified with reasonable accuracy from structural brain imaging data, in particular if they are characterized by specific atrophy patterns, and (ii) that DNN perform better than SVM.

\section{Methods}

\section{Subjects and Demographic Characteristics}

The study included multi-centric data from 940 subjects, i.e. 124 healthy controls and 816 patients from the German Research Consortium of Frontotemporal Lobar Degeneration (www.ftld.de ${ }^{24}$ ) and from the German Atypical Parkinson Consortium Study Group. ${ }^{18,25}$ The patient cohort consisted of 72 patients with AD, 146 patients with bvFTD, 26 patients with CBS, 30 patients with lvPPA, 21 patients with MSA-C, 60 patients with MSA-P, 58 patients with nfvPPA, 203 patients with PD, 154 patients with PSP, and 46 patients with svPPA.

\section{[Figure 1], [Table 1]}


Figure 1 and Table 1 provide an overview over age and gender distribution of the study cohort. Age distribution was compared with the Kruskal-Wallis test and post hoc with a Wilcoxon ranksum test between all pairs of samples (Bonferroni-corrected). Patients with AD were significantly older than patients with bvFTD $(\mathrm{p}<0.05)$. Patients with PSP were significantly older than healthy controls ( $\mathrm{p}<0.001)$ and patients with MSA-C $(\mathrm{p}<0.05)$, MSA-P $(\mathrm{p}<0.001)$, PD $(\mathrm{p}<$ 0.05), bvFTD ( $\mathrm{p}<0.001)$, and svPPA ( $\mathrm{p}<0.001)$. Furthermore, patients with bvFTD were significantly younger than patients with nfvPPA $(\mathrm{p}<0.001)$. Also, patients with svPPA were significantly younger than patients with nfvPPA $(\mathrm{p}<0.05)$.

Gender distribution was tested pairwise with the Fisher test (Bonferroni-corrected) post hoc if the Chi Square test indicated significant differences $($ Chi-square $=38.855, \mathrm{p}<0.001)$. The gender distribution significantly differed between patients with bvFTD and PD ( $<<0.001)$, MSA-P (p < 0.05), and PSP $(\mathrm{p}<0.05)$. Furthermore, there was a significant difference in gender distribution between patients with PD and svPPA $(\mathrm{p}<0.05)$.

The study was conducted according to the Declaration of Helsinki. It was approved by the local ethics committees of all participating centers. Patients, participants, caregivers, or legal representatives gave written informed consent for the study.

\section{Imaging Acquisition and Analysis}

Structural MRI head scans were acquired multi-centrically at German University hospitals. Every subject obtained a T1-weighted three-dimensional (1 mm isovoxel resolution) magnetization prepared rapid gradient echo (MPRAGE) head MRI brain scan. ${ }^{26,24}$ The MPRAGE sequence was converted to ANALYZE 7.5 format and the file names were pseudonymized before further 
processing. There was no sequence adjustment or homogenization between the centers. Instead, the MPRAGE sequence from clinical routine at each center was used. Atlas-based volumetric analysis of the MPRAGE sequence data was done using the LONI Probabilistic Brain Atlas $(\text { LPBA40 })^{27}$ and further masks were derived from this atlas. A detailed description of all image processing steps and atlas structures can be found in ${ }^{18}$. Before being used as predictive features, all volume results were corrected for intracranial volume (ICV).

\section{Training and Evaluation of Classifiers}

In order to reduce the bias of the existing sampling distribution, we used a 5-fold cross validation with the full dataset (models were trained on $80 \%$ of the data ( 4 folds), $20 \%$ served for testing (1 fold)). The folds were selected randomly, and the experiments were repeated ten times. Thus, we trained and evaluated 50 models of each type. In each training iteration, we optimized the learning and hyperparameters of the RF model, the GB, and the SVM using a Bayesian optimization. ${ }^{28}$ During the optimization, overfitting was reduced by using a 5 -fold cross validation, where the training data was split into training and validation data. The optimization was run for 120 iterations.

The kernel applied in the SVM algorithm is critical for its way of functioning. Therefore, the learning and hyperparameters to be optimized also depend on the selected kernel. For this reason, the optimization of the SVM was done separately with linear, sigmoid, polynomial, and radial basis function kernels. Based on this comparison, we considered only SVM with a linear kernel. The linear kernel provided the best SVM performance, where $\mathrm{c}$ is optimized for avoiding misclassifications. 
For the RF classifier we optimized the maximum depth of the tree, the number of features to consider, the minimum number of samples required for a leaf node, and the minimum number of samples required to split an internal node. In case of GB optimization, we additionally optimized the learning rate.

We used a feed forward DNN with 72 neurons in the input layer and 90 neurons in each of the two hidden layers. In order to prevent overfitting we used a dropout rate ${ }^{29}$ of 45 percent for the neurons of the hidden layers and early stopping. ${ }^{30}$ For the weight update we used Adam ${ }^{31}$ and categorical cross entropy, where the optimizer was initialized with $\alpha=0.001, \beta_{1}=0.9=, \beta_{2}=$ 0.999 and $\epsilon=10^{-8}$. The training was done batchwise with samples of 30 patients. More complex architectures have provided worse or similar classification results in the experiments.

The evaluation was done classwise considering the recall, precision, and the F1-score. We dispense with a consideration of the overall recall, overall precision, and the overall F-score, because of the included bias in the actual distribution, which limits the suitability of the F1-score for the model evaluation. ${ }^{32}$ Therefore, this metric is not used for the overall model evaluation, but to show the distribution of predicted classes. To reduce this bias and evaluate the model performance, we use the Cohen's kappa coefficient $\kappa,{ }^{33}$ which is defined as

$$
\kappa=\frac{y-\hat{y}}{1-\hat{y}}
$$

where $y$ denotes the ground truth syndrome classification and $\hat{y}$ the predicted syndrome. In addition, we show the accuracy, which allows a more humanly interpretable evaluation, but without considering the class imbalance. The accuracy is calculated for the total number of elements $n$ in the test fold and has the form:

$$
\operatorname{accuracy}(y, \hat{y})=\frac{1}{n}-1(y, \hat{y})
$$


To better understand the classification process of each model, we used a novel technique, namely "Local Interpretable Model-agnostic Explanations" (LIME), which explains the relationship between the components (here: brain regions) that are used for the classification and its predicted class (here: the syndrome). ${ }^{34}$ This method allowed the direct comparison of the decision process of all four models.

\section{Results}

In the following, the model performance is presented with its classification results and the features, i.e. the brain regions, that have the highest value for the classification decision.

\section{[Figure 2], [Table 1]}

\section{Comparison of Classification Models}

The parameter settings for the tree-based methods (i.e., GB and RF) were found through the optimization processes after around 80 iterations, while the c configuration for the SVM with linear kernel required only around 20 iterations. In the training of the DNN, the validation loss decreased until convergence, which was reached after about 100 epochs on average. The training is stopped, if the validation score was stable for 20 epochs. The convergence and the subsequent overfitting on the training data is shown in Figure 2.

Model-wise performance measures can be found in Table 1. Among the models evaluated in this study, the DNN rendered the best classification results producing a Cohen's Kappa Score slightly larger than 0.4 as well as a total model accuracy of approximately 0.5 . The second-best performance was obtained with SVM, followed by GB and RF. Furthermore, the variability over 
50 permutations was lowest for DNNs, which is reflected by the lowest standard deviation. This indicates that DNN models have the highest reliability of the models across different simulations.

[Table 2]

Modelwise performance measures are shown separately for each of the classes, i.e. diseases, in Table 2. Whereas some diseases such as PSP, svPPA, MSA-P, bvFTD, PD reached relatively high classification performance, other classes reached middle values, i.e. healthy controls and $\mathrm{AD}$, others relatively low performance such as lvPPA, MSA-C and nfvPPA. Of note, $\mathrm{CBS}$ was characterized by lowest performance results. The order of modelwise performance quality across the whole cohort $(\mathrm{DNN}>\mathrm{SVM}>\mathrm{GB}>\mathrm{RF})$ was also observed for AD and bvFTD, whereas the other classes showed a more complex picture.

\section{Importance of Brain Regions}

The LIME method allowed us to assess the contribution of each brain region for classifying each syndrome within a model. An entire listing with the weighting of all brain regions for all models is publically available in the project repository. In the interest of greater clarity, we display the five most important brain regions for all models for three selected pathologies with well-known atrophy pattern (i.e., AD, PSP, and svPPA; see Table 3). Note that the weighting of brain regions was averaged over all patients that were classified correctly by the respective model. All models independently identified the key regions, such as the midbrain for PSP, the inferior temporal gyrus on the left side for svPPA, and the hippocampus for AD. 


\section{Discussion}

In this work we compared several well-established machine learning algorithms (i.e., DNN, GB, $\mathrm{RF}$, and SVM) to predict the diagnosis out of numerous different neurodegenerative syndromes on the basis of pre-structured, atlas-based volumetric brain MRI data. In agreement with our hypothesis, we show that neurodegenerative diseases can be classified from structural brain imaging data, in particular if they are characterized by specific atrophy patterns. Here DNN showed a moderate performance, whereas the three other models showed a fair performance according to Cohen's Kappa scores. Although reasonable for this ambitious clinical question, results were not reaching substantial or even perfect classification results as achieved in comparisons of single neurodegenerative diseases vs. controls. ${ }^{35,36,37,38,39,40}$. This important difference between the diagnostic (disease vs. control) and differential diagnostic (disease vs. disease) approach might be related to etiological overlap between clinical syndromes, unspecific atrophy patterns for some diseases, and even the fact that single patients might show different syndromes in the course of disease. These severe limitations, to be addressed in future studies, hamper the translation of multi-syndrome classifiers to clinical settings to date. In the following, we will discuss our results in more detail.

\section{Structuring Imaging Data for Machine Learning Approaches}

Pre-structuring of the data with atlas-based volumetry had some clear advantages such as easy assessment of particular brain regions as contributing factors for the diagnosis on an individual level as well as across syndromes, thereby increasing the interpretability of the respective model. Moreover, data could be normalized individually by adjusting to the subject's intracranial volume. Presumably, atlas-based volumetry seems to be also superior to voxel-based 
morphometry, because the impact of different centers, scanner types, protocols and applied parameters seem to be decreased by processing steps in atlas-based volumetry-a hypothesis that has to be validated in future studies. Further, using volumetry data also allowed for the training of a model on a single CPU core and with 6 GB RAM. In contrast to this, the training of a

convolutional neural network $(\mathrm{CNN})$ with raw imaging data, ${ }^{41}$ which is the state-of-the-art method for image classification, requires machines with at least one 12 GB GPU or in case of 3D MRI volumes a server with several GPUs. ${ }^{42}$ Finally, pre-structuring of the data increased the anonymity of the data - a general benefit that facilitates central data aggregation without risking the exposure of privacy sensitive medical information.

The reason we were not able to conduct the same experiment with raw imaging data was that we did not have access to the raw images. Despite all the advantages of pre-structured imaging data listed above, it precludes the possibility of data augmentation of raw imaging data - a powerful strategy to increase the amount of training data and thereby boosting model performance.

Furthermore, and perhaps more importantly, predefined feature extraction might lead to a loss of valuable information, which is a clear limitation of our study.

\section{Comparison of Machine Learning Models}

Corresponding to the literature, ${ }^{43}$ our results indicate that the DNN with a simple feedforward architecture is the superior method for this kind of classification task, closely followed by the SVM as illustrated in Table 2. While neural networks became the state-of-the-art method for the processing of imaging data and text data, $\mathrm{DNNs}^{44}$ were shown to outperform tree-based methods as well as SVM with structured data.However, it is informative to take a closer look at model performance and model robustness for each single class individually, especially considering the 
size of the class and the specificity of atrophy patterns respectively (see Table 3 ). The DNN performed best (high F1-score and high robustness) in large classes (e.g., PD, bvFTD, AD, and PSP) where there was a sufficient data for the model loss to converge. Generally, classes with smaller sample size expectedly led to models with weaker performance measures. GB and SVM seemed to best perform for smaller classes (e.g., MSA-C, lvPPA, CBS), while RF rendered the best robustness for smaller classes. The high robustness of RF in this case might be due to prediction ensembles, while the superior performance of GB and SVM over the DNN might reflect those models possibly needing less data than neural networks. Notably, classes with more specific atrophy patterns (e.g., svPPA and AD) were also best predicted by the DNN despite the comparatively small sample size possible due to faster convergence of the loss function. As expected, diseases with regionally specific and pronounced atrophy patterns such as svPPA, AD and PSP were generally better classified than diseases with wide-spread and rather weak atrophy such as CBS (see Figure 3). The confusion matices in Figure 3 give a nice overview over the class-specific performance of the different methods and nicely show that the DNN has a reasonable performace for all classes.

In conclusion, the larger the dataset the better the performance. It was here, where the DNNs were able to clearly show their superiority with respect to classification performance as well as robustness. However, the point of convergence is the critical factor for good performance. For this, a balanced validation set must be used.

\section{Validation}

The validation was not only performed by using the prediction score, but also the standard deviation of the prediction scores as a measure of robustness. Generally, the standard deviation of the model performance depends on the training data set used, which is why we chose k-fold cross 
validation $^{45}$ instead of a leave-one-out cross validation. In contrast to a leave-one-out cross validation, a k-fold cross validation changes the class distribution in the training data set over the different experiments, which affects the model training. When a leave-one-out cross validation is performed, a class imbalance in the data set always exists in a similar ratio (with the exception of the validation instance) and is therefore reflected in a lower model quality. The highest overall robustness was observed for the DNN while the ensemble methods in turn were least robust, possibly due to their general propensity to overfit the models.

\section{Model Performance}

Both recall and precision are class-wise measures and are therewith independent of the number of true negatives, which are over-represented in a multiclass problem and thereby inflate measures contingent on the true negatives. The F1-score is a combination of both, precision and recall and is supposed to give a more holistic measure of class-wise model performance.

For overall model performance accuracy is a popular measure, which we included in the reported metrics. However, in the case of a multiclass problem with large imbalance, accuracy is not able to provide an honest reflection of the overall model performance. For this reason, we limit the consideration to the Cohen's Kappa score for the overall model evaluation (Table 2), because this score allows a normalization by the size of the respective class. ${ }^{33}$ For the interpretation of the Cohen's Kappa score the following scheme can be used: $0-0.20$ as slight, $0.21-0.40$ as fair, $0.41-0.60$ as moderate, $0.61-0.80$ as substantial, and $0.81-1$ as almost perfect agreement. ${ }^{46}$ According to this scheme every DNN performs with a moderate performance and the three other models with a fair performance. The confusion matrix (Figure 3) further visualizes how the DNN 
performs better for all different classes in comparison to the tree-based methods, where the model overfits towards the larger classes such as Parkinson, PSP, and bvFTD.

\section{Feature Importance}

To better understand the process of decision making of every model, we extracted the feature importance with the LIME method. Despite the differences in performance metrics, all methods were able to reproduce well known atrophy patterns of respective syndromes. Note that unlike in binary disease-vs-healthy classification tasks, the interpretation of the feature importance resulting from a multiclass classification problem is more ambiguous. The "important features" listed above merely reflect which brain regions were most important to differentiate the respective diagnosis from all other diagnoses included in the classification task.

\section{Limitations}

While the use of volumetry data simplifies the task of classification, it simultaneously limits the classification basis to atrophy patterns only and excludes brain tissue that has no effect on atrophy. The two-stage approach consisting of the volumetry calculation and the classification of the diseases also carries the risk of error summation, which can lead to increased prediction error compared to approaches that are using the original data.

\section{Conclusion}

In conclusion we found the DNN to be the best method to assess imaging derived structured data. However, the performance of different methods largely depends on the dataset and the underlying classification problem. To select the optimal method, one should test and validate several methods and consider the available computing resources. Despite the mentioned advantages of 
pre-structuring brain data, our future work will extend the application of $\mathrm{CNN}^{41}$ on raw MRI data as well, for which remarkable results have previously been achieved for the diagnosis of smaller numbers of neurodegenerative diseases. ${ }^{47,48,49,50,51,52}$ This addresses the aforementioned limitations, however, challenges arise in data privacy when processing the data and in the increased demand of training data, which requires further data acquisition considering the rarity of coverage of the various neurodegenerative syndromes. 


\section{References}

1. Woo CW, Chang LJ, Lindquist MA, Wager TD. Building better biomarkers: brain models in translational neuroimaging.. Nat Neurosci. 2017;20:365-377.

2. Laakso MP, Soininen H, Partanen K, et al. MRI of the hippocampus in Alzheimer's disease: sensitivity, specificity, and analysis of the incorrectly classified subjects.. Neurobiol Aging. $1998 ; 19: 23-31$.

3. Schroeter ML, Neumann J. Combined Imaging Markers Dissociate Alzheimer's Disease and Frontotemporal Lobar Degeneration - An ALE Meta-Analysis.. Front Aging Neurosci. 2011;3:10.

4. Albrecht F, Bisenius S, Morales SR, Neumann J, Schroeter ML. Disentangling the neural correlates of corticobasal syndrome and corticobasal degeneration with systematic and quantitative ALE meta-analyses.. NPJ Parkinsons Dis. 2017;3:12.

5. Albrecht F, Bisenius S, Neumann J, Whitwell J, Schroeter ML. Atrophy in midbrain \& cerebral/cerebellar pedunculi is characteristic for progressive supranuclear palsy - A doublevalidation whole-brain meta-analysis.. Neuroimage Clin. 2019;22:101722.

6. Bisenius S, Neumann J, Schroeter ML. Response to the letter on 'Validating new diagnostic imaging criteria for primary progressive aphasia via anatomical likelihood estimation metaanalyses'.. Eur J Neurol. 2016;23:e52-3.

7. Schroeter ML, Stein T, Maslowski N, Neumann J. Neural correlates of Alzheimer's disease and mild cognitive impairment: a systematic and quantitative meta-analysis involving 1351 patients.. Neuroimage. 2009;47:1196-1206. 
8. Schroeter ML, Laird AR, Chwiesko C, et al. Conceptualizing neuropsychiatric diseases with multimodal data-driven meta-analyses - the case of behavioral variant frontotemporal dementia.. Cortex. 2014;57:22-37.

9. Gorno-Tempini ML, Hillis AE, Weintraub S, et al. Classification of primary progressive aphasia and its variants.. Neurology. 2011;76:1006-1014.

10. McKhann GM, Knopman DS, Chertkow H, et al. The diagnosis of dementia due to Alzheimer's disease: recommendations from the National Institute on Aging-Alzheimer's Association workgroups on diagnostic guidelines for Alzheimer's disease.. Alzheimers Dement. 2011;7:263-269.

11. Rascovsky K, Hodges JR, Knopman D, et al. Sensitivity of revised diagnostic criteria for the behavioural variant of frontotemporal dementia.. Brain. 2011;134:2456-2477.

12. Albrecht F, Ballarini T, Neumann J, Schroeter ML. FDG-PET hypometabolism is more sensitive than MRI atrophy in Parkinson's disease: A whole-brain multimodal imaging metaanalysis.. Neuroimage Clin. 2019;21:101594.

13. Dwyer DB, Falkai P, Koutsouleris N. Machine Learning Approaches for Clinical Psychology and Psychiatry.. Annu Rev Clin Psychol. 2018;14:91-118.

14. Arbabshirani MR, Plis S, Sui J, Calhoun VD. Single subject prediction of brain disorders in neuroimaging: Promises and pitfalls.. Neuroimage. 2017;145:137-165.

15. Pellegrini E, Ballerini L, Hernandez MDCV, et al. Machine learning of neuroimaging for assisted diagnosis of cognitive impairment and dementia: A systematic review.. Alzheimers Dement (Amst). 2018;10:519-535. 
16. Dukart J, Mueller K, Barthel H, Villringer A, Sabri O, Schroeter ML. Meta-analysis based SVM classification enables accurate detection of Alzheimer's disease across different clinical centers using FDG-PET and MRI.. Psychiatry Res. 2013;212:230-236.

17. Bisenius S, Mueller K, Diehl-Schmid J, et al. Predicting primary progressive aphasias with support vector machine approaches in structural MRI data. Neuroimage Clin. 2017;14:334-343.

18. Huppertz HJ, Möller L, Südmeyer M, et al. Differentiation of neurodegenerative parkinsonian syndromes by volumetric magnetic resonance imaging analysis and support vector machine classification.. Mov Disord. 2016;31:1506-1517.

19. LeCun Y, Bengio Y, Hinton G. Deep learning.. Nature. 2015;521:436-444.

20. Yu KH, Zhang C, Berry GJ, et al. Predicting non-small cell lung cancer prognosis by fully automated microscopic pathology image features.. Nat Commun. 2016;7:12474.

21. Esteva A, Kuprel B, Novoa RA, et al. Dermatologist-level classification of skin cancer with deep neural networks.. Nature. 2017;542:115-118.

22. Fauw JD, Ledsam JR, Romera-Paredes B, et al. Clinically applicable deep learning for diagnosis and referral in retinal disease. Nat Med. 2018;24:1342-1350.

23. Raczko E, Zagajewski B. Comparison of support vector machine random forest and neural network classifiers for tree species classification on airborne hyperspectral APEX images. European Journal of Remote Sensing. 2017;50(1):144-154.

doi:10.1080/22797254.2017.1299557

24. Otto M, Ludolph AC, Landwehrmeyer B, et al. [German consortium for frontotemporal lobar degeneration]. Nervenarzt. 2011;82:1002-1005. 
25. Möller L, Kassubek J, Südmeyer M, et al. Manual MRI morphometry in Parkinsonian syndromes.. Mov Disord. 2017;32:778-782.

26. Brant-Zawadzki M, Gillan GD, Nitz WR. MP RAGE: a three-dimensional T1-weighted, gradient-echo sequence-initial experience in the brain.. Radiology. 1992;182(3):769-775. doi:10.1148/radiology.182.3.1535892

27. Shattuck DW, Mirza M, Adisetiyo V, et al. Construction of a 3D probabilistic atlas of human cortical structures.. Neuroimage. 2008;39:1064-1080.

28. Frazier PI. A Tutorial on Bayesian Optimization. arxiv. 2018;arXiv:1807.02811 [stat.ML]. https://arxiv.org/abs/1807.02811v1.

29. Srivastava N, Hinton G, Krizhevsky A, Sutskever I, Salakhutdinov R. Dropout: A Simple Way to Prevent Neural Networks from Overfitting. Journal of Machine Learning Research. 2014;15. http://jmlr.org/papers/v15/srivastava14a.html.

30. Prechelt L. Automatic early stopping using cross validation: quantifying the criteria.. Neural Netw. 1998;11:761-767.

31. Kingma DP, Ba J. Adam: A Method for Stochastic Optimization. arxiv. 2015;arXiv:1412.6980 [cs.LG]. https://arxiv.org/abs/1412.6980v9.

32. Powers DMW. What the F-measure doesn't measure: Features, Flaws, Fallacies and Fixes. arxiv. 2015;arXiv:1503.06410 [cs.IR]. https://arxiv.org/abs/1503.06410v2.

33. Cohen J. A Coefficient of Agreement for Nominal Scales. Educational and Psychological Measurement. 1960;20(1):37-46. doi:10.1177/001316446002000104 
34. Ribeiro MT, Singh S, Guestrin C. Why Should I Trust You?: Explaining the Predictions of Any Classifier. arxiv. 2016;arXiv:1602.04938 [cs.LG]. https://arxiv.org/abs/1602.04938v3.

35. Woo CW, Chang LJ, Lindquist MA, Wager TD. Building better biomarkers: brain models in translational neuroimaging. Nat Neurosci. 2017 Feb 23;20(3):365-377. doi: 10.1038/nn.4478

36. Albrecht F, Mueller K, Ballarini T, et al. Unraveling corticobasal syndrome and alien limb syndrome with structural brain imaging. Cortex. 2019 Aug;117:33-40. doi:

10.1016/j.cortex.2019.02.015.

37. Ballarini T, Albrecht F, Mueller K, et al. Disentangling brain functional network remodeling in corticobasal syndrome - A multimodal MRI study. Neuroimage Clin. 2020;25:102112. doi: 10.1016/j.nicl.2019.102112.

38. Bisenius S, Mueller K, Diehl-Schmid J, et al. Predicting primary progressive aphasias with support vector machine approaches in structural MRI data. Neuroimage Clin. 2017 Feb 6;14:334343. doi: 10.1016/j.nicl.2017.02.003.

39. Meyer S, Mueller K, Stuke K, et al. Predicting behavioral variant frontotemporal dementia with pattern classification in multi-center structural MRI data. Neuroimage Clin. 2017 Feb 6;14:656-662. doi: 10.1016/j.nicl.2017.02.001. eCollection 2017. PMID: 28348957 Free PMC article.

40. Mueller K, Jech R, Bonnet C, et al. Disease-Specific Regions Outperform Whole-Brain Approaches in Identifying Progressive Supranuclear Palsy: A Multicentric MRI Study. Front Neurosci. 2017 Mar 7;11:100. doi: 10.3389/fnins.2017.00100. eCollection 2017.

41. Krizhevsky A, Sutskever I, Hinton GE. ImageNet classification with deep convolutional neural networks. Communications of the ACM. 2017;60(6):84-90. doi:10.1145/3065386 
42. Wen J, Thibeau-Sutre E, Diaz-Melo M, et al. Convolutional Neural Networks for Classification of Alzheimer's Disease: Overview and Reproducible Evaluation. arxiv. 2019; arXiv:1904.07773 [cs.LG]. https://arxiv.org/abs/1904.07773v3.

43. Huang GB, Chen YQ, Babri HA. Classification ability of single hidden layer feedforward neural networks.. IEEE Trans Neural Netw. 2000;11:799-801.

44. Bengio Y, Grandvalet Y. Bias in Estimating the Variance of K-Fold Cross-Validation. In: Statistical Modeling and Analysis for Complex Data Problems. Springer-Verlag; :75-95. doi:10.1007/0-387-24555-3_5

45. Landis JR, Koch GG. The measurement of observer agreement for categorical data. Biometrics. 1977;33:159-174.

46. Farooq A, Anwar SM, Awais M, Rehman S. A deep CNN based multi-class classification of Alzheimers disease using MRI. In: 2017 IEEE International Conference on Imaging Systems and Techniques (IST). IEEE; 2017. doi:10.1109/ist.2017.8261460

47. Sarraf S, DeSouza DD, Anderson J, Tofighi G. DeepAD: Alzheimer's Disease Classification via Deep Convolutional Neural Networks using MRI and fMRI. August 2016. doi:10.1101/070441

48. Yuan L, Wei X, Shen H, Zeng LL, Hu D. Multi-Center Brain Imaging Classification Using a Novel 3D CNN Approach. IEEE Access. 2018;6:49925-49934.

doi:10.1109/access.2018.2868813

49. Basaia S, Agosta F, Wagner L, et al. Automated classification of Alzheimer's disease and mild cognitive impairment using a single MRI and deep neural networks.. Neuroimage Clin. 2019;21:101645. 
50. Tapiola T, Pennanen C, Tapiola M, et al. MRI of hippocampus and entorhinal cortex in mild cognitive impairment: a follow-up study.. Neurobiol Aging. 2008;29:31-38.

51. Albrecht F, Mueller K, Ballarini T, et al. Unraveling corticobasal syndrome and alien limb syndrome with structural brain imaging.. Cortex. 2019;117:33-40.

52. Yves G, Yoshua B. Entropy Regularization. In: Semi-Supervised Learning. The MIT Press; 2006:151-168. doi:10.7551/mitpress/9780262033589.003.0009 


\section{Tables}

\begin{tabular}{|c|c|c|c|}
\hline & $\mathrm{n}$ & Age (years) & Gender (female/male) \\
\hline $\mathrm{AD}$ & 72 & $66.67(+/-9.59)$ & $39 / 33$ \\
\hline bvFTD & 146 & $61.68(+/-9.67)$ & $53 / 93$ \\
\hline CBS & 26 & $65.96(+/-6.91)$ & $15 / 11$ \\
\hline lvPPA & 30 & $67.33(+/-5.60)$ & $13 / 17$ \\
\hline MSA-C & 21 & $63.05(+/-7.24)$ & $11 / 10$ \\
\hline MSA-P & 60 & $63.29(+/-7.99)$ & $38 / 22$ \\
\hline nfvPPA & 58 & $68.46(+/-8.32)$ & $29 / 29$ \\
\hline PD & 203 & $64.08(+/-11.21)$ & $135 / 68$ \\
\hline PSP & 154 & $69.03(+/-6.47)$ & $82 / 72$ \\
\hline svPPA & 46 & $62.14(+/-8.31)$ & $19 / 27$ \\
\hline Healthy controls & 124 & $63.71(+/-10.00)$ & $60 / 64$ \\
\hline
\end{tabular}

Table 1 Demographic characteristics for patients and healthy controls

Note: Data are reported as mean \pm standard deviation. Abbreviations: AD Alzheimer's disease; bvFTD behavioral variant frontotemporal dementia; CBS corticobasal syndrome; lvPPA logopenic variant primary progressive aphasia; MSA-C multiple system atrophy (cerebellar dysfunction subtype); MSA-P multiple system atrophy (parkinsonian subtype); nfvPPA nonfluent variant primary progressive aphasia; PD Parkinson's disease; PSP progressive supranuclear palsy; svPPA semantic variant primary progressive aphasia. 


$$
\text { RF } \quad \text { GB } \quad \text { SVM }
$$

Cohen's Kappa $\quad 0.325 \pm 0.036 \quad 0.358 \pm 0.036 \quad 0.383 \pm 0.043 \quad 0.404 \pm 0.03$

$\begin{array}{lllll}\text { Accuracy } & 0.429 \pm 0.032 & 0.456 \pm 0.032 & 0.472 \pm 0.038 & 0.496 \pm 0.025\end{array}$

Table 2 Metrics for model comparison. Note: Data are reported as mean \pm standard deviation.

Abbreviations: DNN deep neural network; GB gradient boosting; RF random forest; SVM support vector machine. 


\begin{tabular}{|c|c|c|c|c|}
\hline Class & Model & Precision & Recall & F1-Score \\
\hline \multirow{4}{*}{$\mathrm{AD}$} & RF & $0.285 \pm 0.138$ & $0.228 \pm 0.112$ & $0.236 \pm 0.101$ \\
\hline & GB & $0.351 \pm 0.186$ & $0.246 \pm 0.126$ & $0.276 \pm 0.13$ \\
\hline & SVM & $0.368 \pm 0.148$ & $0.43 \pm 0.114$ & $0.385 \pm 0.114$ \\
\hline & DNN & $0.391 \pm 0.138$ & $0.436 \pm 0.135$ & $0.391 \pm 0.099$ \\
\hline \multirow{4}{*}{ bvFTD } & $\mathrm{RF}$ & $0.405 \pm 0.109$ & $0.4 \pm 0.09$ & $0.396 \pm 0.084$ \\
\hline & GB & $0.406 \pm 0.095$ & $0.434 \pm 0.089$ & $0.415 \pm 0.08$ \\
\hline & SVM & $0.453 \pm 0.092$ & $0.436 \pm 0.094$ & $0.439 \pm 0.077$ \\
\hline & DNN & $0.526 \pm 0.11$ & $0.491 \pm 0.089$ & $0.499 \pm 0.073$ \\
\hline \multirow{4}{*}{ CBS } & $\mathrm{RF}$ & $0.054 \pm 0.207$ & $0.016 \pm 0.058$ & $0.023 \pm 0.085$ \\
\hline & GB & $0.127 \pm 0.257$ & $0.046 \pm 0.102$ & $0.064 \pm 0.131$ \\
\hline & SVM & $0.083 \pm 0.188$ & $0.047 \pm 0.105$ & $0.058 \pm 0.129$ \\
\hline & DNN & $0.047 \pm 0.177$ & $0.073 \pm 0.024$ & $0.026 \pm 0.095$ \\
\hline \multirow{4}{*}{ lvPPA } & $\mathrm{RF}$ & $0.164 \pm 0.303$ & $0.058 \pm 0.103$ & $0.077 \pm 0.13$ \\
\hline & GB & $0.305 \pm 0.331$ & $0.151 \pm 0.169$ & $0.185 \pm 0.194$ \\
\hline & SVM & $0.274 \pm 0.304$ & $0.178 \pm 0.197$ & $0.185 \pm 0.172$ \\
\hline & DNN & $0.228 \pm 0.348$ & $0.111 \pm 0.180$ & $0.128 \pm 0.185$ \\
\hline \multirow{4}{*}{ MSA-C } & RF & $0.202 \pm 0.331$ & $0.123 \pm 0.199$ & $0.140 \pm 0.219$ \\
\hline & GB & $0.368 \pm 0.392$ & $0.179 \pm 0.203$ & $0.227 \pm 0.243$ \\
\hline & SVM & $0.38 \pm 0.324$ & $0.328 \pm 0.308$ & $0.304 \pm 0.241$ \\
\hline & DNN & $0.313 \pm 0.448$ & $0.136 \pm 0.241$ & $0.176 \pm 0.278$ \\
\hline \multirow{4}{*}{ MSA-P } & $\mathrm{RF}$ & $0.511 \pm 0.176$ & $0.395 \pm 0.134$ & $0.433 \pm 0.134$ \\
\hline & GB & $0.541 \pm 0.166$ & $0.405 \pm 0.125$ & $0.453 \pm 0.127$ \\
\hline & SVM & $0.538 \pm 0.149$ & $0.458 \pm 0.17$ & $0.48 \pm 0.142$ \\
\hline & DNN & $0.554 \pm 0.162$ & $0.425 \pm 0.162$ & $0.466 \pm 0.141$ \\
\hline \multirow{4}{*}{ nfvPPA } & $\mathrm{RF}$ & $0.164 \pm 0.307$ & $0.042 \pm 0.074$ & $0.060 \pm 0.103$ \\
\hline & GB & $0.250 \pm 0.233$ & $0.113 \pm 0.094$ & $0.146 \pm 0.120$ \\
\hline & SVM & $0.242 \pm 0.183$ & $0.107 \pm 0.087$ & $0.139 \pm 0.1$ \\
\hline & DNN & $0.226 \pm 0.289$ & $0.060 \pm 0.074$ & $0.087 \pm 0.101$ \\
\hline \multirow{4}{*}{$\mathrm{PD}$} & RF & $0.410 \pm 0.070$ & $0.610 \pm 0.098$ & $0.484 \pm 0.058$ \\
\hline & GB & $0.431 \pm 0.057$ & $0.642 \pm 0.100$ & $0.511 \pm 0.274$ \\
\hline & SVM & $0.485 \pm 0.065$ & $0.605 \pm 0.093$ & $0.533 \pm 0.058$ \\
\hline & DNN & $0.465 \pm 0.062$ & $0.744 \pm 0.097$ & $0.567 \pm 0.055$ \\
\hline \multirow{4}{*}{ PSP } & $\mathrm{RF}$ & $0.625 \pm 0.091$ & $0.674 \pm 0.081$ & $0.644 \pm 0.065$ \\
\hline & GB & $0.652 \pm 0.081$ & $0.694 \pm 0.081$ & $0.669 \pm 0.061$ \\
\hline & SVM & $0.615 \pm 0.024$ & $0.661 \pm 0.083$ & $0.629 \pm 0.295$ \\
\hline & DNN & $0.646 \pm 0.084$ & $0.673 \pm 0.085$ & $0.653 \pm 0.060$ \\
\hline \multirow{4}{*}{ svPPA } & $\mathrm{RF}$ & $0.539 \pm 0.123$ & $0.566 \pm 0.165$ & $0.533 \pm 0.102$ \\
\hline & GB & $0.591 \pm 0.144$ & $0.585 \pm 0.173$ & $0.570 \pm 0.125$ \\
\hline & SVM & $0.652 \pm 0.135$ & $0.59 \pm 0.165$ & $0.602 \pm 0.118$ \\
\hline & DNN & $0.567 \pm 0.120$ & $0.698 \pm 0.149$ & $0.614 \pm 0.105$ \\
\hline \multirow{4}{*}{ Healthy controls } & $\mathrm{RF}$ & $0.362 \pm 0.104$ & $0.374 \pm 0.088$ & $0.354 \pm 0.006$ \\
\hline & GB & $0.386 \pm 0.125$ & $0.368 \pm 0.095$ & $0.364 \pm 0.079$ \\
\hline & SVM & $0.438 \pm 0.106$ & $0.448 \pm 0.093$ & $0.436 \pm 0.078$ \\
\hline & DNN & $0.450 \pm 0.133$ & $0.357 \pm 0.141$ & $0.375 \pm 0.099$ \\
\hline
\end{tabular}

Table 3 Class-wise performance metrics for multi-syndrome classification 
Abbreviations: AD Alzheimer's disease; bvFTD behavioral variant frontotemporal dementia; CBS corticobasal syndrome; lvPPA logopenic variant primary progressive aphasia; MSA-C multiple system atrophy (cerebellar dysfunction subtype); MSA-P multiple system atrophy (parkinsonian subtype); nfvPPA nonfluent variant primary progressive aphasia; PD Parkinson's disease; PSP progressive supranuclear palsy; svPPA semantic variant primary progressive aphasia. 


\begin{tabular}{|c|c|c|c|c|}
\hline & $\mathrm{RF}$ & GB & SVM & DNN \\
\hline \multirow{6}{*}{$\mathrm{AD}$} & $\begin{array}{l}\text { Hippocampus and } \\
\text { Amygdala left }\end{array}$ & $\begin{array}{l}\text { Hippocampus and } \\
\text { Amygdala left }\end{array}$ & $\begin{array}{l}\text { Inferior temporal } \\
\text { gyrus left }\end{array}$ & $\begin{array}{l}\text { Inferior temporal } \\
\text { gyrus left }\end{array}$ \\
\hline & $\begin{array}{l}\text { Hippocampus and } \\
\text { Amygdala right }\end{array}$ & $\begin{array}{l}\text { Hippocampus and } \\
\text { Amygdala right }\end{array}$ & $\begin{array}{l}\text { Hippocampus and } \\
\text { Amygdala left }\end{array}$ & $\begin{array}{l}\text { Midbrain tegmen- } \\
\text { tum plane }\end{array}$ \\
\hline & $\begin{array}{l}\text { Midbrain tegmen- } \\
\text { tum plane }\end{array}$ & $\begin{array}{l}\text { Fusiform gyrus } \\
\text { left }\end{array}$ & $\begin{array}{l}\text { Fusiform gyrus } \\
\text { left }\end{array}$ & $\begin{array}{l}\text { Middle temporal } \\
\text { gyrus left }\end{array}$ \\
\hline & $\begin{array}{l}\text { Fusiform gyrus } \\
\text { left }\end{array}$ & $\begin{array}{l}\text { Postcentral gyrus } \\
\text { right }\end{array}$ & $\begin{array}{l}\text { Angular gyrus } \\
\text { right }\end{array}$ & Midbrain plane \\
\hline & $\begin{array}{l}\text { Middle temporal } \\
\text { gyrus left }\end{array}$ & $\begin{array}{l}\text { Middle temporal } \\
\text { gyrus left }\end{array}$ & $\begin{array}{l}\text { Hippocampus and } \\
\text { Amygdala right }\end{array}$ & $\begin{array}{l}\text { Middle temporal } \\
\text { gyrus left }\end{array}$ \\
\hline & $\mathrm{RF}$ & GB & SVM & DNN \\
\hline \multirow{6}{*}{ PSP } & $\begin{array}{l}\text { Midbrain tegmen- } \\
\text { tum plane }\end{array}$ & $\begin{array}{l}\text { Midbrain tegmen- } \\
\text { tum plane }\end{array}$ & $\begin{array}{l}\text { Midbrain tegmen- } \\
\text { tum plane }\end{array}$ & $\begin{array}{l}\text { Midbrain tegmen- } \\
\text { tum plane }\end{array}$ \\
\hline & Midbrain plane & Midbrain plane & Midbrain plane & Midbrain plane \\
\hline & Brainstem right & Brainstem right & Brainstem left & Brainstem right \\
\hline & $\begin{array}{l}\text { Postcentral gyrus } \\
\text { left }\end{array}$ & $\begin{array}{l}\text { Postcentral gyrus } \\
\text { left }\end{array}$ & Brainstem right & Brainstem left \\
\hline & Brainstem left & Brainstem left & $\begin{array}{ll}\text { Inferior } & \text { frontal } \\
\text { gyrus left } & \end{array}$ & $\begin{array}{ll}\text { Inferior } & \text { frontal } \\
\text { gyrus left } & \end{array}$ \\
\hline & $\mathrm{RF}$ & GB & SVM & DNN \\
\hline \multirow{5}{*}{ svPPA } & $\begin{array}{l}\text { Inferior temporal } \\
\text { gyrus left }\end{array}$ & $\begin{array}{l}\text { Inferior temporal } \\
\text { gyrus left }\end{array}$ & $\begin{array}{l}\text { Inferior temporal } \\
\text { gyrus left }\end{array}$ & $\begin{array}{l}\text { Inferior temporal } \\
\text { gyrus left }\end{array}$ \\
\hline & $\begin{array}{l}\text { Middle temporal } \\
\text { gyrus left }\end{array}$ & $\begin{array}{l}\text { Middle temporal } \\
\text { gyrus left }\end{array}$ & $\begin{array}{l}\text { Middle temporal } \\
\text { gyrus left }\end{array}$ & $\begin{array}{l}\text { Middle temporal } \\
\text { gyrus left }\end{array}$ \\
\hline & $\begin{array}{l}\text { Hippocampus and } \\
\text { Amygdala left }\end{array}$ & $\begin{array}{l}\text { Parahippocampal } \\
\text { gyrus left }\end{array}$ & $\begin{array}{l}\text { Fusiform gyrus } \\
\text { left }\end{array}$ & $\begin{array}{l}\text { Fusiform gyrus } \\
\text { left }\end{array}$ \\
\hline & $\begin{array}{l}\text { Midbrain tegmen- } \\
\text { tum plane }\end{array}$ & $\begin{array}{l}\text { Fusiform gyrus } \\
\text { left }\end{array}$ & $\begin{array}{l}\text { Parahippocampal } \\
\text { gyrus left }\end{array}$ & $\begin{array}{l}\text { Hippocampus and } \\
\text { Amygdala left }\end{array}$ \\
\hline & Midbrain plane & $\begin{array}{ll}\begin{array}{l}\text { Superior } \\
\text { gyrus left }\end{array} & \end{array}$ & $\begin{array}{l}\text { Inferior temporal } \\
\text { gyrus right }\end{array}$ & $\begin{array}{l}\text { Superior temporal } \\
\text { gyrus left }\end{array}$ \\
\hline
\end{tabular}

Table 4 Brain regions with highest weighting, i.e. importance, for classification.

Abbreviations: AD Alzheimer's disease; DNN deep neural network; GB gradient boosting; RF random forest; SVM support vector machine; PSP progressive supranuclear palsy; svPPA semantic variant primary progressive aphasia. 


\section{Figures}

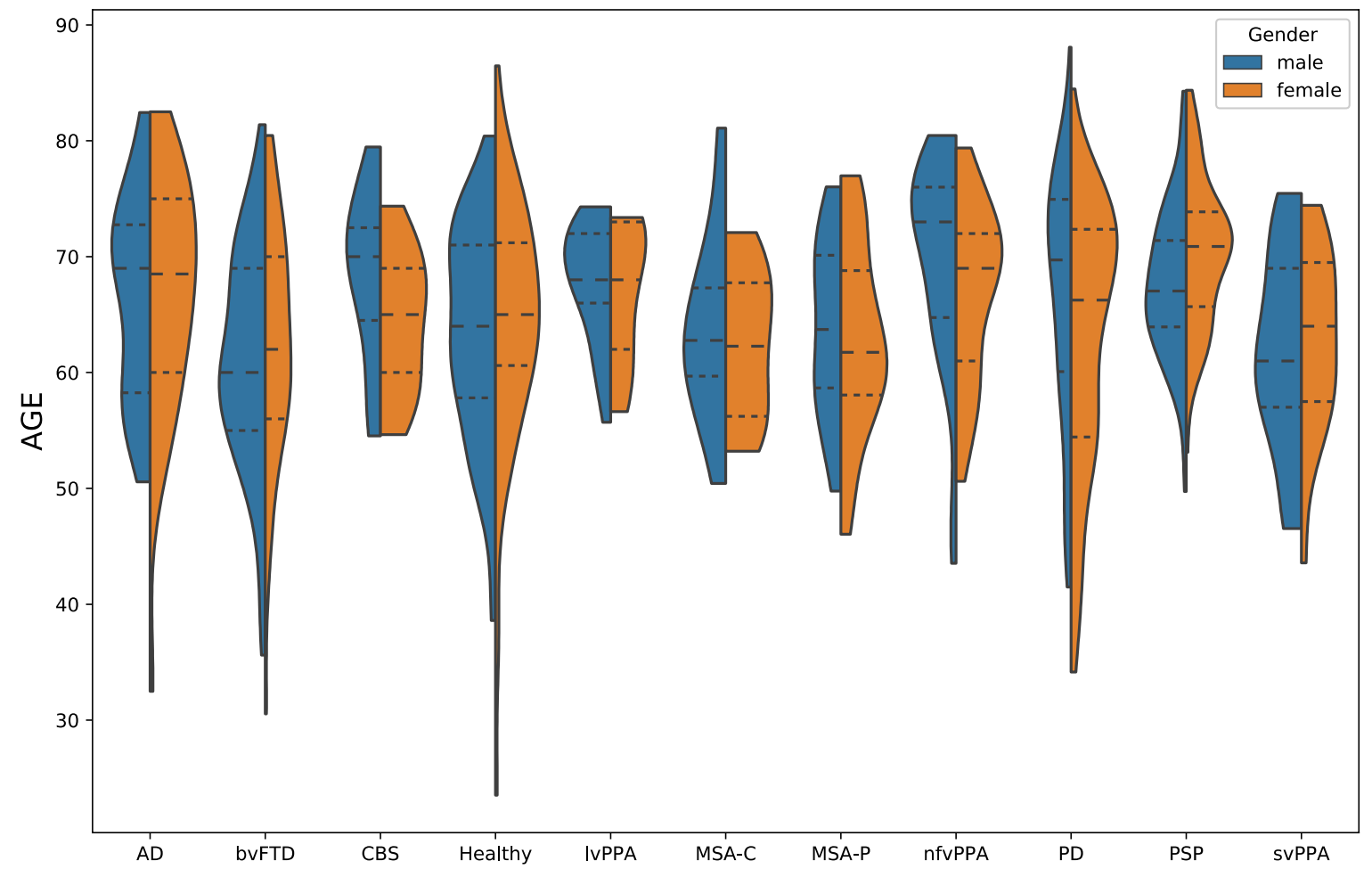

Figure 1 Violin plot of the age and gender distribution of the cohort sample. The dashed line indicates the mean and the dotted line the standard deviation.

Abbreviations: AD Alzheimer's disease; bvFTD behavioral variant frontotemporal dementia; CBS corticobasal syndrome; lvPPA logopenic variant primary progressive aphasia; MSA-C multiple system atrophy (cerebellar dysfunction subtype); MSA-P multiple system atrophy (parkinsonian subtype); nfvPPA nonfluent variant primary progressive aphasia; PD Parkinson's disease; PSP progressive supranuclear palsy; svPPA semantic variant primary progressive aphasia. 


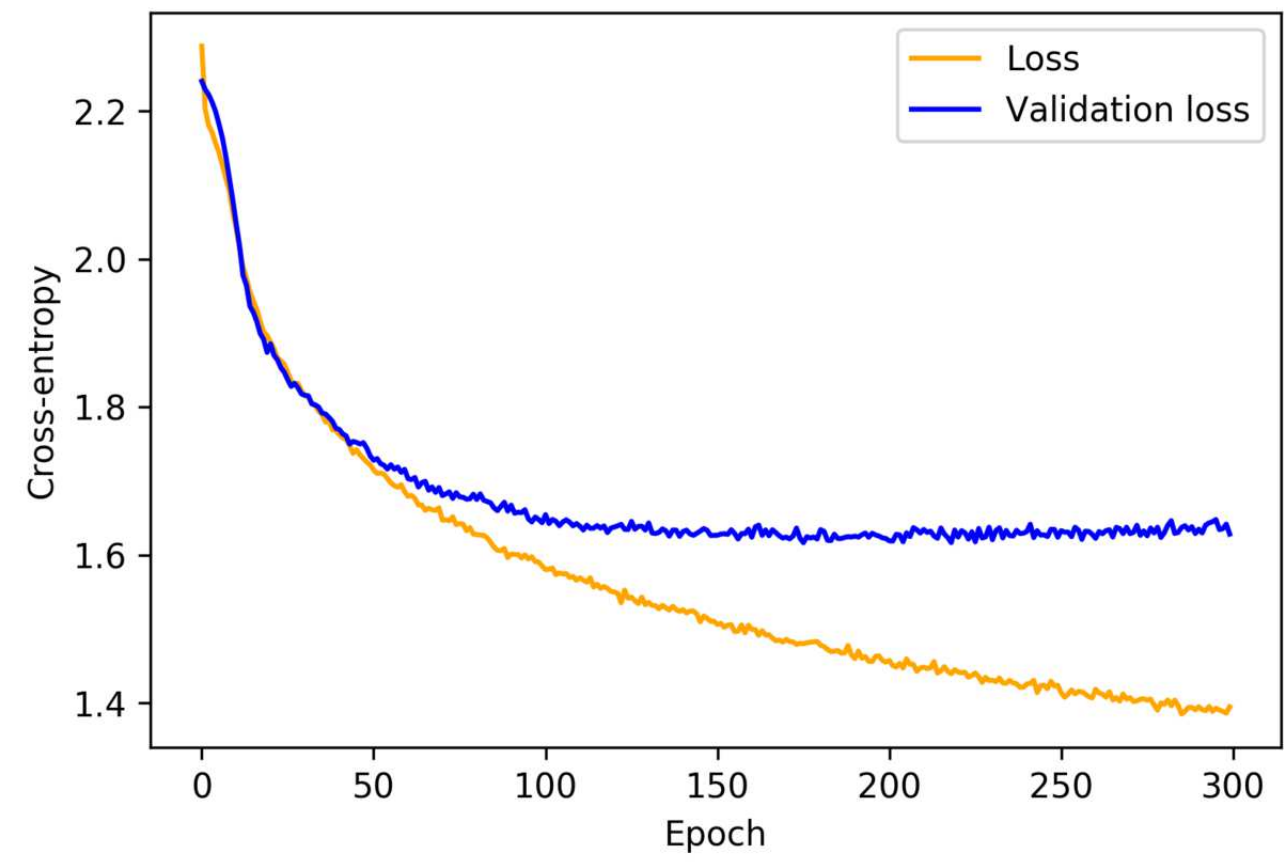

Figure 2 Averaged training loss and validation loss of the DNN. For the consideration of overfitting, the early stopping was dispensed with for this recording. 

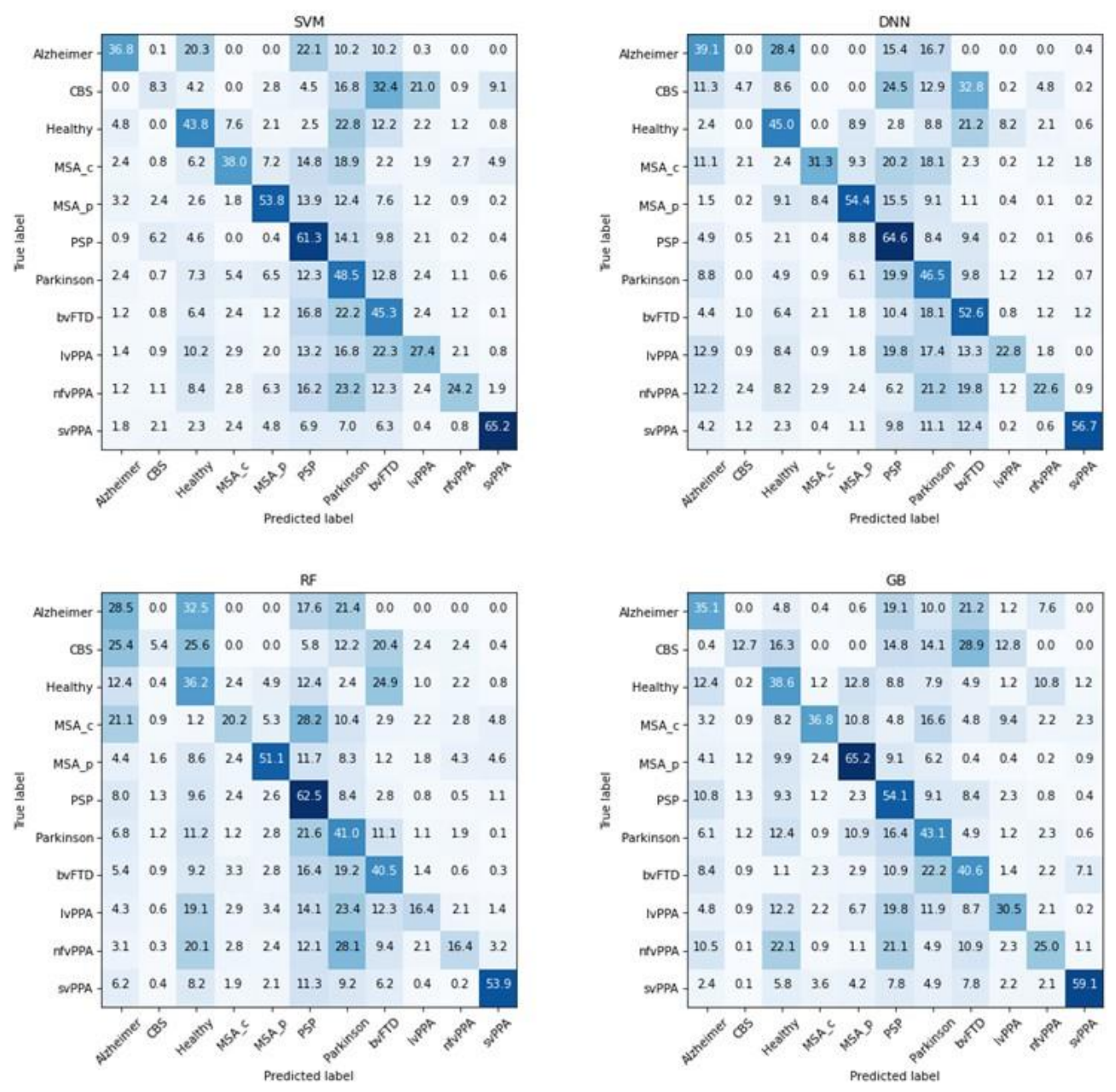

Figure 3 Confusion matrix for every classification model. The values are averaged over all folds and figured as percentage value of the true label. The coloration depends on the number of predicted cases in percent (the scale goes from the highest value (dark blue) to the value 0 (white)). The confusion matrix shows row normalized percentages, which results in precision is shown for matching classes in column and row. 


\section{Declarations}

\section{Ethical Approval and Consent to participate}

n.a.

\section{Consent for publication}

All authors read and approved the final manuscript.

\section{Availability of data and materials}

Project repository: https://github.com/Leoniela/Comparison-ML-Algorithms-Neurodegen

\section{Competing interests}

The authors declare that they have no conflict of interest.

\section{Funding}

This work was supported by the German Federal Ministry of Education, and Research (BMBF) by a grant given to the German FTLD Consortium (FKZ O1GI1007A), by the German Research Foundation DFG (SCHR 774/5-1), by the Parkinson's Disease Foundation (PDF-IRG-1307) and by the Michael J. Fox Foundation (MJFF-11362). Günter Höglinger was funded by the Deutsche Forschungsgemeinschaft (DFG, German Research Foundation) under Germany's Excellence Strategy within the framework of the Munich Cluster for Systems Neurology (EXC 2145 SyNergy - ID 390857198), and the NOMIS foundation (FTLD project).

\section{Authors' contributions}

LL, SN and MLS designed the research study and analytical approaches. AM, SAS, KFs, KFl, HJ, JK, ML, JP, AS, MSy, AD, JDS, MO, KE, EH, RHR, AS, MSü, WO, JK, GH contributed data.

LL, SN, HJH, JR, KM, AV and MLS contributed essentially to data analysis and interpretation. LL, SN wrote the first draft of the manuscript, revised by MLS. All authors contributed to the paper and agreed to the final version of the manuscript.

\section{Acknowledgements}

See Funding section.

\section{Authors' information}

Leonie Lampe, MD

AICURA medical GmbH, Berlin

Clinic for Cognitive Neurology, University Clinic Leipzig, Germany

Max Planck Institute for Human Cognitive and Brain Sciences, Leipzig, Germany

Sebastian Niehaus, M.Sc.

AICURA medical GmbH, Berlin 
Carl Gustav Carus Faculty of Medicine, Institute for Medical Informatics and Biometry, TU Dresden, Dresden, Germany

Hans-Jürgen Huppertz, MD

Swiss Epilepsy Clinic, Klinik Lengg, Zurich, Switzerland

Alberto Merola, $\mathrm{PhD}$

AICURA medical GmbH, Berlin

Janis Reinelt

AICURA medical GmbH, Berlin

Karsten Mueller, $\mathrm{PhD}$

Max Planck Institute for Human Cognitive and Brain Sciences, Leipzig, Germany

Sarah Anderl-Straub, $\mathrm{PhD}$

Department of Neurology, University of Ulm, Germany

Klaus Fassbender, MD

Department of Neurology, Saarland University, Homburg, Germany

Klaus Fliessbach, MD

Clinic for Neurodegenerative Diseases and Geriatric Psychiatry, University of Bonn, and

German Center for Neurodegenerative Diseases (DZNE), Bonn, Germany

Holger Jahn, MD

Clinic for Psychiatry and Psychotherapy, University Hospital Hamburg-Eppendorf, Germany

Johannes Kornhuber, MD

Department of Psychiatry and Psychotherapy, Friedrich-Alexander-University of Erlangen-

Nuremberg, Erlangen, Germany

Martin Lauer, MD

Department of Psychiatry and Psychotherapy, University Wuerzburg, Germany

Johannes Prudlo, MD

Department of Neurology, University of Rostock, and DZNE, Rostock, Germany

Anja Schneider, MD

Clinic for Neurodegenerative Diseases and Geriatric Psychiatry, University of Bonn, and German Center for Neurodegenerative Diseases (DZNE), Bonn, Germany

Matthis Synofzik, MD

Department of Neurodegenerative Diseases, Centre for Neurology \& Hertie-lnstitute for Clinical Brain Research, University of Tuebingen, Germany \& DZNE, Tuebingen, Germany

Adrian Danek, MD 
Department of Neurology, Ludwig-Maximilians-Universität München, Germany

Janine Diehl-Schmid, MD

Department of Psychiatry and Psychotherapy, Technical University of Munich, Germany

Markus Otto, MD

Department of Neurology, University of Ulm, Germany

FTLD-Consortium Germany

Arno Villringer, MD2,3;

Clinic for Cognitive Neurology, University Clinic Leipzig, Germany

Max Planck Institute for Human Cognitive and Brain Sciences, Leipzig, Germany

Karl Egger, MD

Department of Neuroradiology, University Hospital of Freiburg, Germany

Elke Hattingen, MD

Department of Neuroradiology, University Hospital of Frankfurt, Germany

Rüdiger Hilker-Roggendorf, MD

Department of Neurology, Klinikum Vest, Recklinghausen, Germany

Alfons Schnitzler, MD

Institute of Clinical Neurosciences and Medical Psychology, Heinrich Heine University of Düsseldorf, Germany

Martin Südmeyer, MD

Institute of Clinical Neurosciences and Medical Psychology, Heinrich Heine University of Düsseldorf, Germany

Department of Neurology, Ernst von Bergmann Klinikum, Potsdam, Germany

Wolfgang Oertel, MD

Department of Neurology, Philips-University Marburg, Germany

German Atypical Parkinson Consortium Study Group

Jan Kassubek, MD

Department of Neurology, University of Ulm, Germany

Günter Höglinger, MD

Department of Neurology, Technical University of Munich, Germany; German Center for Neurodegenerative Diseases, Munich, Germany

Matthias L Schroeter, MD PhD 
Clinic for Cognitive Neurology, University Clinic Leipzig, Germany 


\section{Figures}

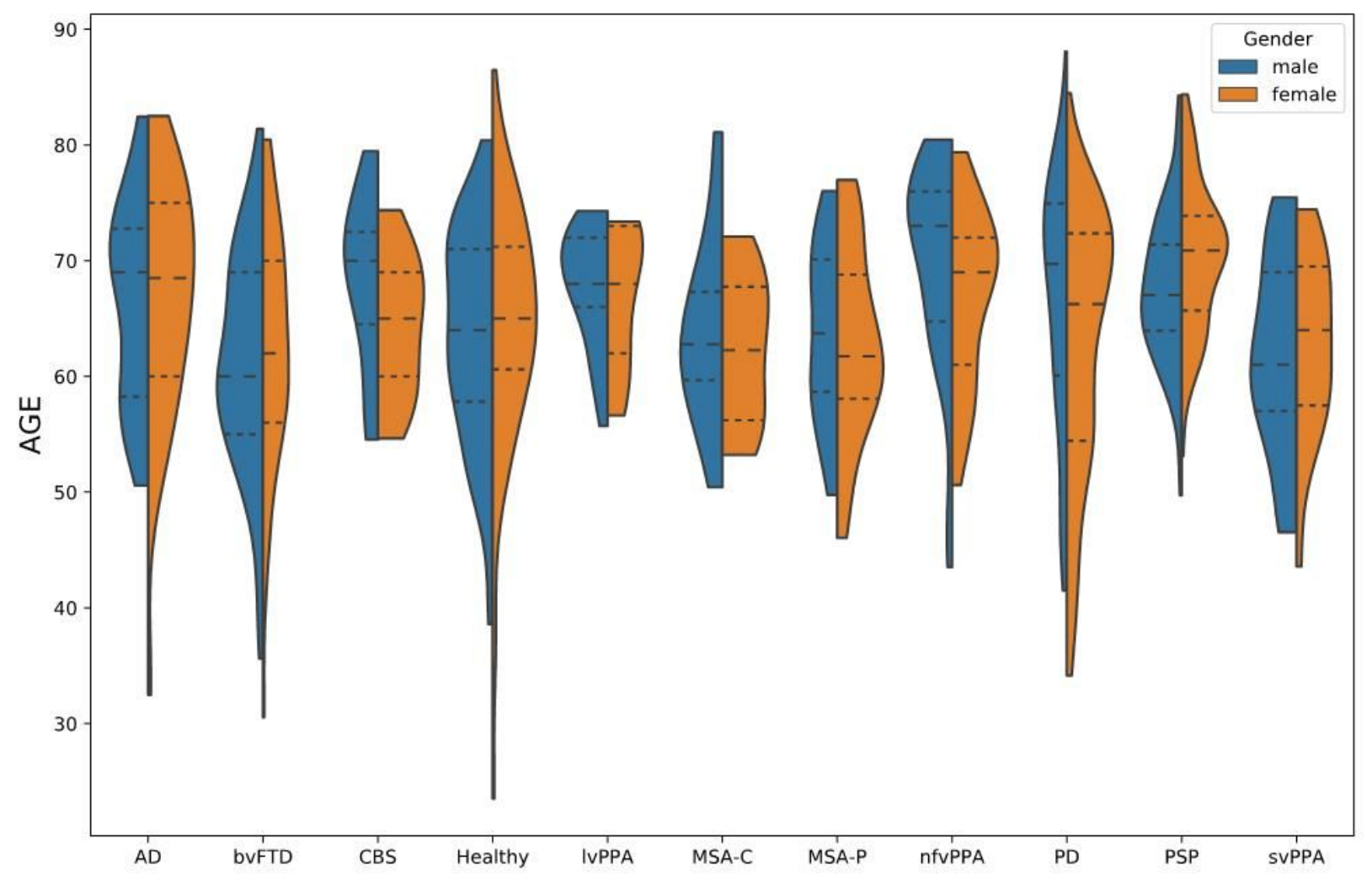

\section{Figure 1}

Violin plot of the age and gender distribution of the cohort sample. The dashed line indicates the mean and the dotted line the standard deviation. Abbreviations: AD Alzheimer's disease; bvFTD behavioral variant frontotemporal dementia; CBS corticobasal syndrome; IVPPA logopenic variant primary progressive aphasia; MSA-C multiple system atrophy (cerebellar dysfunction subtype); MSA-P multiple system atrophy (parkinsonian subtype); nfvPPA nonfluent variant primary progressive aphasia; PD Parkinson's disease; PSP progressive supranuclear palsy; svPPA semantic variant primary progressive aphasia. 


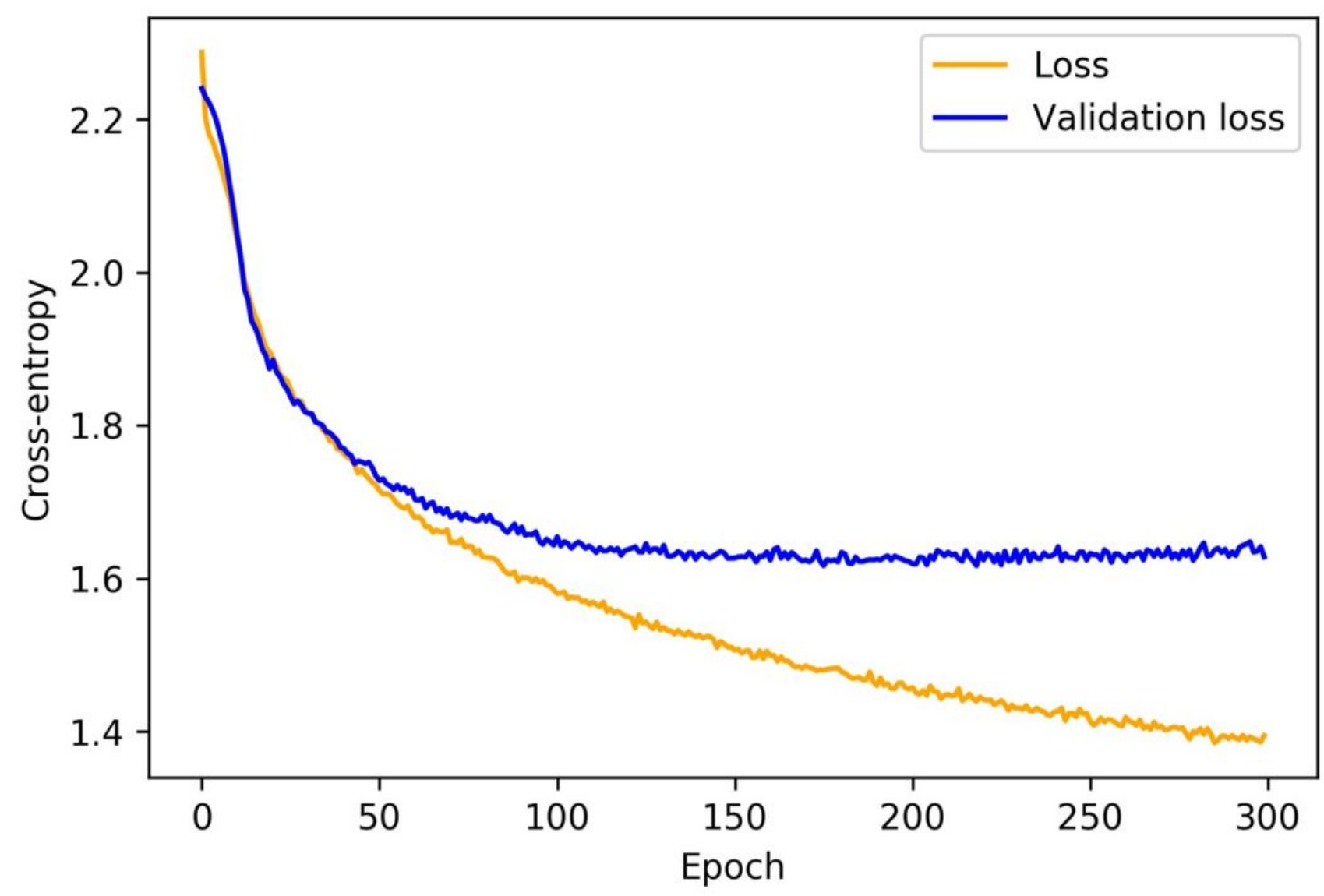

Figure 2

Averaged training loss and validation loss of the DNN. For the consideration of overfitting, the early stopping was dispensed with for this recording. 

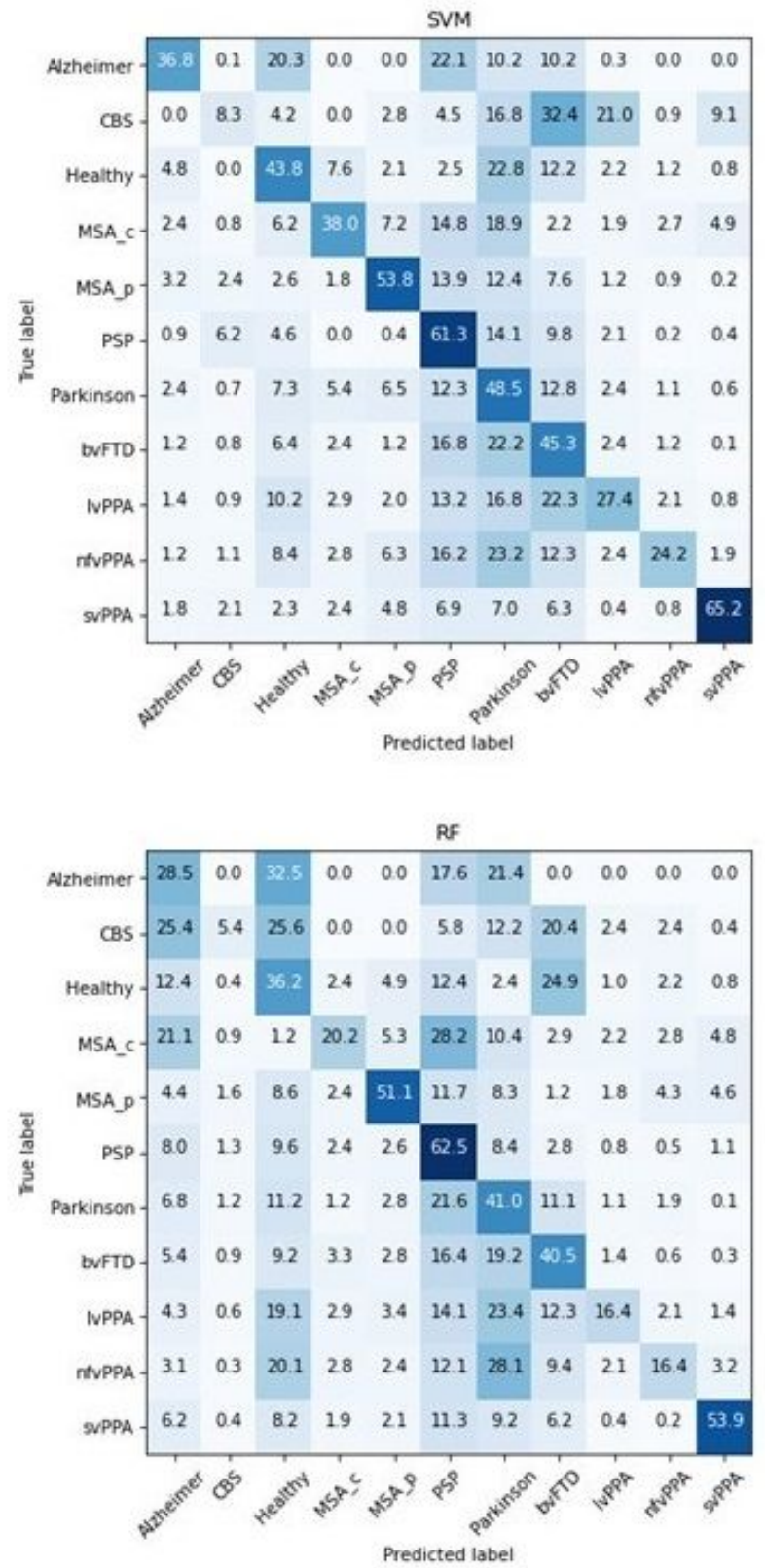
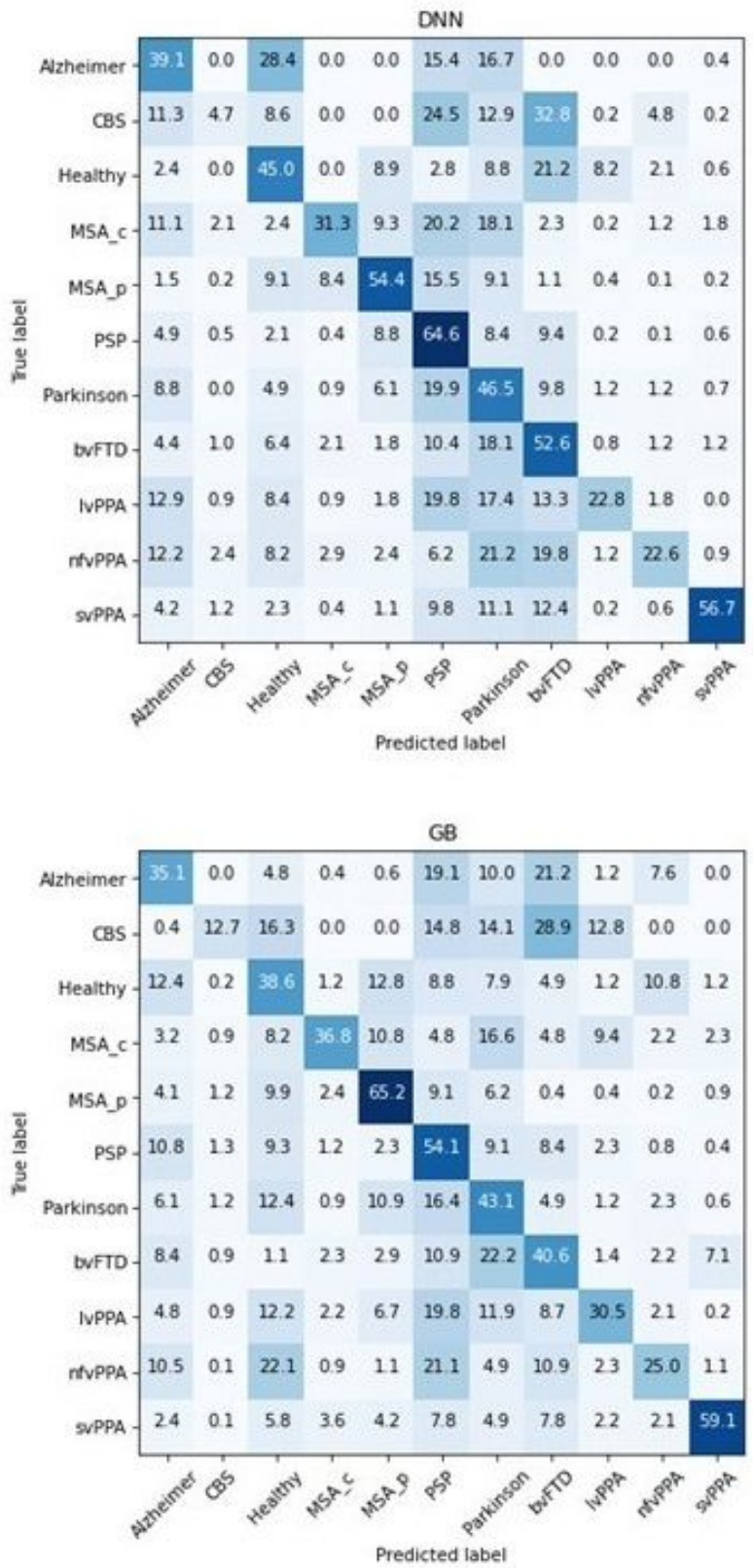

Figure 3

Confusion matrix for every classification model. The values are averaged over all folds and figured as percentage value of the true label. The coloration depends on the number of predicted cases in percent (the scale goes from the highest value (dark blue) to the value 0 (white)). The confusion matrix shows row normalized percentages, which results in precision is shown for matching classes in column and row. 